\title{
New tools for the visualization of glial fibrillary acidic protein in living cells
}

\author{
Ricardo Letra-Vilela ${ }^{1,2, \#}$, Ricardo Quiteres ${ }^{1,2, \#}$, Fernanda Murtinheira ${ }^{1,2}$, Alvaro Crevenna ${ }^{3}$, \\ Zach Hensel $^{2, *}$ and Federico Herrera ${ }^{1,2, *}$ (1) \\ ${ }^{1}$ Cell Structure and Dynamics Laboratory, Faculdade de Ciências, Universidade de Lisboa, Lisbon, Portugal, ${ }^{2}$ Instituto de \\ Tecnologia Quimica e Biologica (ITQB-NOVA), Universidade Nova de Lisboa, Oeiras, Portugal, and EMBL Rome, Epigenetics \\ and Neurobiology Unit, Via E. Ramarini 32, 00015 Monterotondo (RM), Italy. \\ *Corresponding authors: E-mails: fherrera@fc.ul.pt; zach.hensel@itqb.unl.pt \\ \#Equal contribution
}

(Received 02 December 2019; Revised 26 December 2019; Accepted 27 December 2019)

\begin{abstract}
The glial fibrillary acidic protein (GFAP) is an intermediate filament widely used to identify and label astroglial cells, a very abundant and relevant glial cell type in the central nervous system. A major hurdle in studying its behavior and function arises from the fact that GFAP does not tolerate well the addition of protein tags to its termini. Here, we tagged human GFAP (hGFAP) with an enhanced green fluorescent protein (EGFP) for the first time, and substituted a previously reported EGFP tag on mouse GFAP (mGFAP) by a more versatile Halo Tag. Both versions of tagged GFAP were able to incorporate into the normal GFAP filamentous network in glioma cells, and Alexander disease-related mutations or pharmacological disruption of microtubules and actin filaments interfered with GFAP dynamics. These new tools could provide new fruitful venues for the study of GFAP oligomerization, aggregation and dynamics in living cells.
\end{abstract}

Key words: glial fibrillary acidic protein; transposase; Halo Tag; single-molecule; SRRF

\section{Introduction}

The glial fibrillary acidic protein (GFAP) is an intermediate filament widely known as a molecular marker for astroglia (Middeldorp \& Hol, 2011). GFAP expression is strikingly enhanced in conditions of stress, inflammation or injury (Anderson, Ao, \& Sofroniew, 2014; Li et al., 2019; Middeldorp \& Hol, 2011), and mutations in this filament are associated to Alexander disease (Messing, 2018). However, the structure and function of this filament remain mostly unknown. This is due, at least in part, to the intolerance of GFAP to tags at the $\mathrm{N}$ - or $\mathrm{C}$-terminus. Most GFAP studies were restricted to fixed cells and immunocytochemistry (Hsiao et al., 2005; Perng et al., 2008), and early attempts to tag human GFAP (hGFAP) had mixed success, as cells often showed GFAP aggregation or poor filament formation (Bachetti et al., 2008; Perng et al., 2008; Tulyeu et al., 2019). Very recently, mouse GFAP (mGFAP) was successfully tagged with EGFP by introducing a linker between the filament and the fluorescent protein (Mignot et al., 2007). Although mGFAP shares 95\% homology to hGFAP (Anderson et al., 2014; Middeldorp \& Hol, 2011), there are relevant differences that could determine different mechanisms of regulation and function.

\footnotetext{
(C) The Author(s) 2020. This is an Open Access article, distributed under the terms of the Creative Commons Attribution-NonCommercialNoDerivatives licence (http://creativecommons.org/licenses/by-nc-nd/4.0/), which permits non-commercial re-use, distribution, and reproduction in any medium, provided the original work is unaltered and is properly cited. The written permission of Cambridge University Press must be obtained for commercial re-use or in order to create a derivative work.
} 


\section{Objective}

Here, we developed new tools for the study of GFAP behavior and function in living cells. First, we created an EGFP-tagged version of hGFAP where the tag is within the GFAP sequence, and not at its Nor C-terminus, in order to avoid the issues related to terminal GFAP tags (Bachetti et al., 2008; Perng et al., 2008; Tulyeu et al., 2019). Secondly, we used a previously reported mGFAP construct tagged with EGFP at the C-terminus (Mignot et al., 2007) to insert a Halo Tag in substitution of EGFP. The Halo tag is far more versatile than EGFP (England, Luo, \& Cai, 2015) and will allow super-resolution, singlemolecule and protein-protein interaction studies on GFAP.

\section{Methods}

A commercial transposase was used to insert a kanamycin resistance gene and EGFP into the human GFAP sequence in a random manner (dx.doi.org/10.17504/protocols.io.77fhrin). The final selected construct had EGFP inserted after amino acid 183 of the GFAP sequence, but the transposase reaction produced an unavoidable repetition of amino acids 181-183 right after the EGFP sequence, as previously described (Sheridan et al., 2002). EGFP was substituted by a Halo tag in the pEGFP-N3-mGFAP plasmid (dx.doi.org/10.17504/protocols.io.77fhrjn). Single R236H or R239C Alexander disease-related mutations were inserted by site-directed mutagenesis into our mGFAP or hGFAP constructs, respectively (dx. doi.org/10.17504/protocols.io.77fhrjn). Human U251 cells and rat C6 glioma cells were maintained, transiently transfected with the corresponding plasmids and treated as described in dx.doi.org/10.17504/ protocols.io.77ehrje. Imaging and image analysis were carried out as described in dx.doi.org/10.17504/ protocols.io.77ghrjw. Protein extraction and western blotting was carried out as described previously (Herrera et al., 2009). Statistical analysis and graphical representation of data were performed using Sigmaplot software (Systat Software, Inc., San Jose, CA, USA). Sample data are represented as mean \pm standard deviation of at least 3 independent experiments. Statistical significance was evaluated by means of a one-way ANOVA followed by a Tukey's test. Results were considered significant when $\mathrm{p}<0.05$.

\section{Results}

Both EGFP-hGFAP and mGFAP-Halo constructs formed normal filaments in U251 glioma cells, but the mGFAP-Halo construct failed to do so in rat C6 glioma cells (Fig. 1A). They were suitable for obtaining super-resolution-like pictures by means of super-resolution radial fluctuations (SRRF) (Fig. 1B). None of them formed normal fibers in HEK293 cells (Fig. 1D). The site of insertion of EGFP in hGFAP was not suitable for hosting a Halo tag or bimolecular fluorescence complementation tags (Fig. 1C). While the Alexander disease-related mutation R239C induced aggregation of hGFAP in $48 \%$ of cells, the equivalent $\mathrm{R} 236 \mathrm{H}$ mutant mGFAP showed disorganization of fibers with signs of aggregation in only $14 \%$ of cells (Fig. 2A-C). The expression levels of mutant GFAP were similar to their wild type counterparts (Fig. 2E). However, we cannot rule out the possibility that mGFAP-Halo constructs are expressed at lower overall levels than hGFAP-EGFP constructs, which could explain a lower level of aggregation in mutant mGFAP-Halo. The anti-amyloidogenic curcumin derivative CNB-001 (10 $\mu \mathrm{M})(\mathrm{Liu}$, Dargusch, Maher, \& Schubert, 2008) partially prevented hGFAP aggregation (Fig. 2D). Single-molecule analysis of mGFAP-Halo dynamics confirmed that the $\mathrm{R} 236 \mathrm{H}$ mutation produced a higher proportion of freemoving GFAP molecules, and indicated that pharmacological interference with microtubules or actin filaments significantly disrupted mGFAP dynamics (Fig. 3).

\section{Discussion}

Our tagged hGFAP and mGFAP constructs behaved mostly as expected for endogenous GFAP when they were expressed in living glioma cells and challenged with previously known genetic and pharmacological modifiers of GFAP fibrillization. The only anomalies detected are the lack of GFAP aggregates 

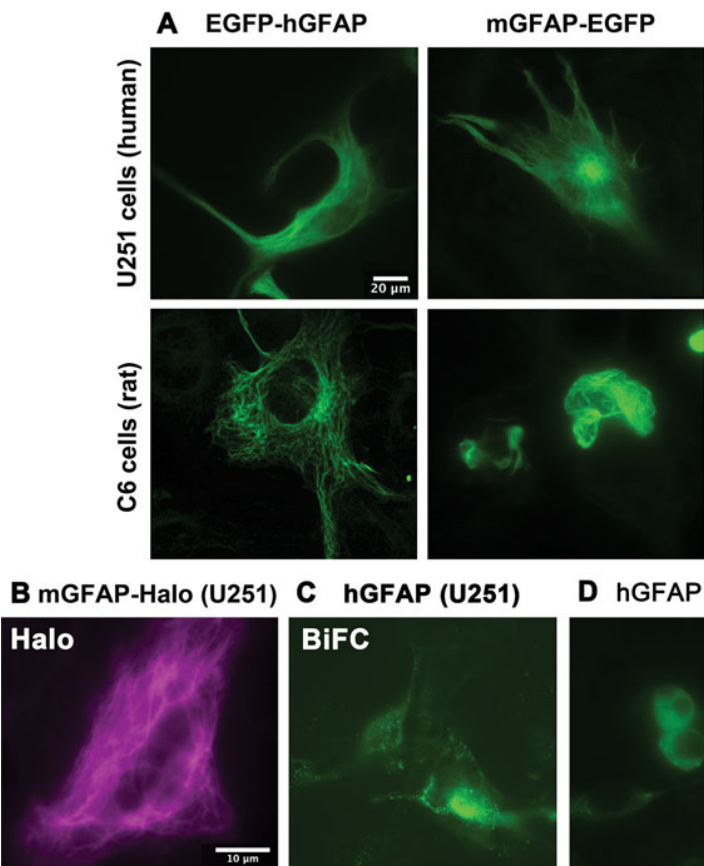

D hGFAP (HEK293)
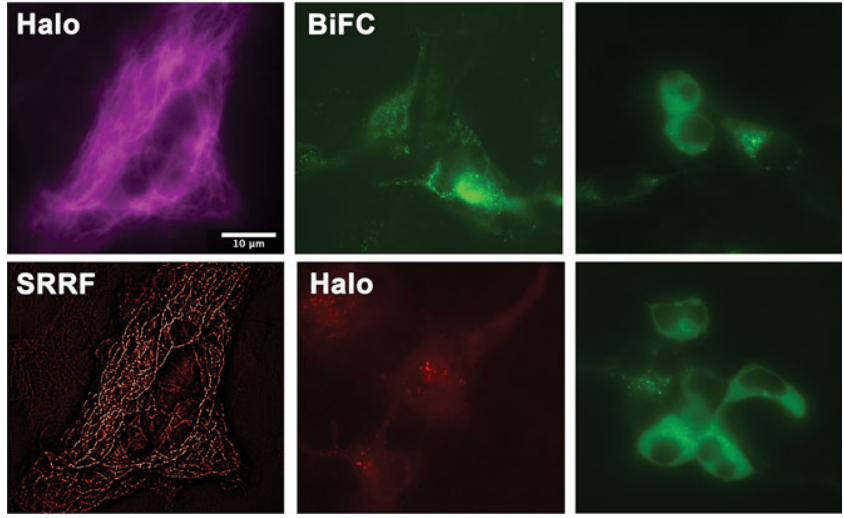

Figure 1. Tagged versions of human and mouse GFAP form normal fibers in glioblastoma cells. Human U251 or rat C6 glioblastoma cells were transiently transfected with different human or mouse GFAP constructs, and imaged $24 \mathrm{~h}$ later. A, When transfected into U251 human glioblastoma cells EGFP-hGFAP and mGFAP-EGFP exhibited a normal GFAP filamentous network. However, when the same constructs were transfected into C6 rat glioma cells, only EGFP-hGFAP formed a regular filamentous network. B, mGFAP-Halo constructs (incubated with the JF549 Halo ligand, $100 \mathrm{nM}$ ) also produced normal filaments only in U251 cells. Widefield images of the mGFAP-Halo construct were further analyzed using the ImageJ software with the NanoJ SRRF plug-in to obtain a more defined image of the intermediate filament network. C, Our attempts to substitute EGFP for bimolecular fluorescence complementation (BiFC) tags Venus 1 (amino acids 1-157) and Venus 2 (amino acids 158-238) or Halo Tag were unsuccessful. These are representative images of U251 cells transfected with these constructs, where residual fluorescence can be observed but has no recognizable pattern (i.e. filaments, bundles or aggregates). D, Transfection of HEK293 cells with the EGFP-hGFAP construct produced either homogenous fluorescence or aggregates, but no filamentous network.

in the R236H mGFAP-Halo mutant and its inability to form fibers in rat glioma cells. These are facts that we cannot currently explain and in which the performance of the EGFP-hGFAP version was superior. Interestingly, the only functional site of EGFP insertion in hGFAP is located in a region (amino acids 183-184) that shows very little frequency of mutations related to Alexander disease (Messing, 2018).

\section{Conclusions}

We reported a successful attempt to EGFP-tag hGFAP and a new version of mGFAP tagged with Halo. The EGFP-hGFAP construct is adaptable to different species and behaves as expected when it is mutated, but it is not suitable for single molecule analysis (although it can be used for super-resolution imaging in combination with the SRRF plug-in (Gustafsson et al., 2016). On the other hand, the mGFAP-Halo 


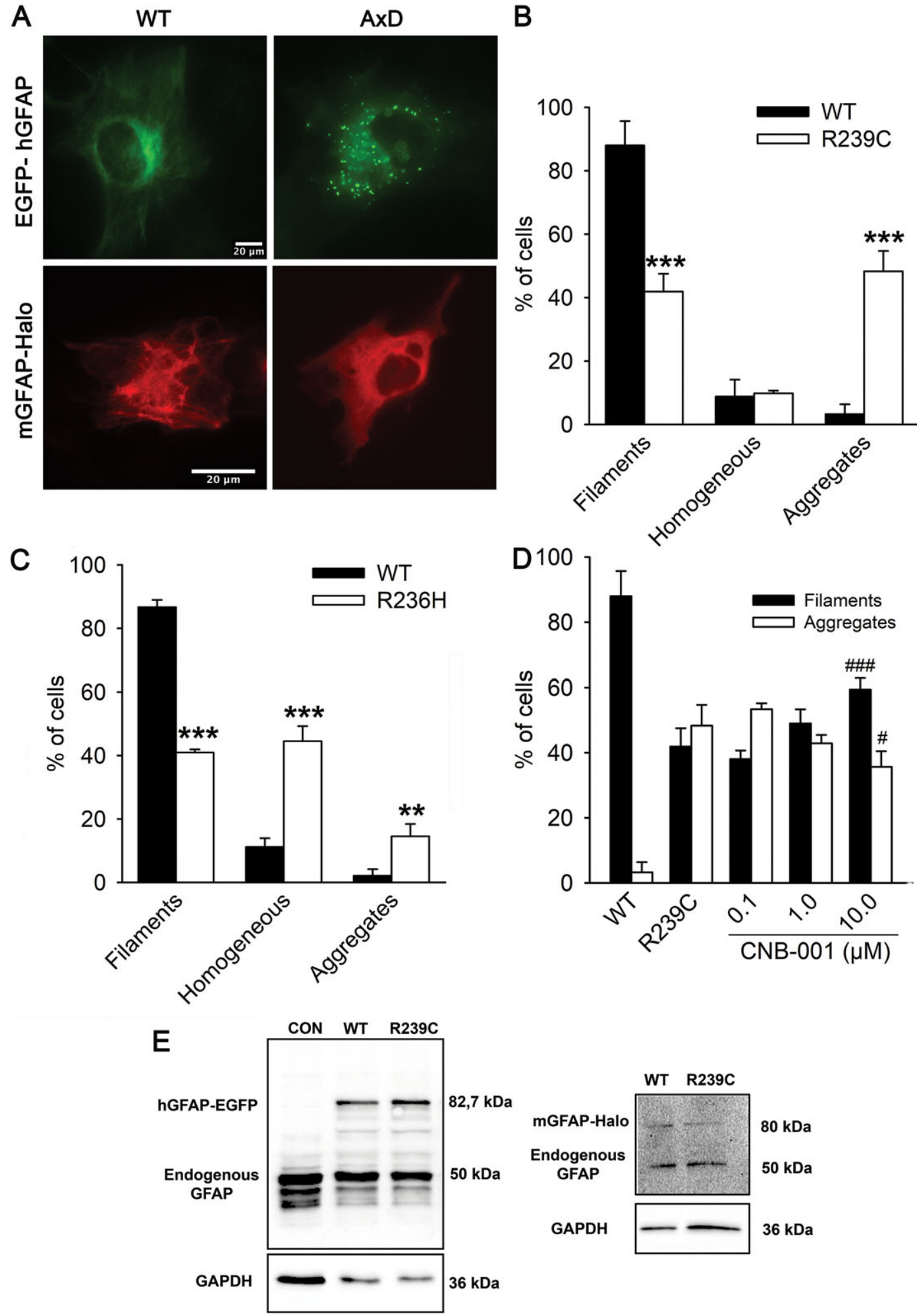

Figure 2. Alexander disease-related mutations cause filament disorganization of GFAP. A, U251 cells were transiently transfected with either wild type (WT) or Alexander disease (AxD)-related versions of EGFP-hGFAP or mGFAP-Halo (R239C or $\mathrm{R} 236 \mathrm{H}$, respectively), and pictures were taken $24 \mathrm{~h}$ later. In the case of the mGFAP-Halo constructs, cells were incubated with the 

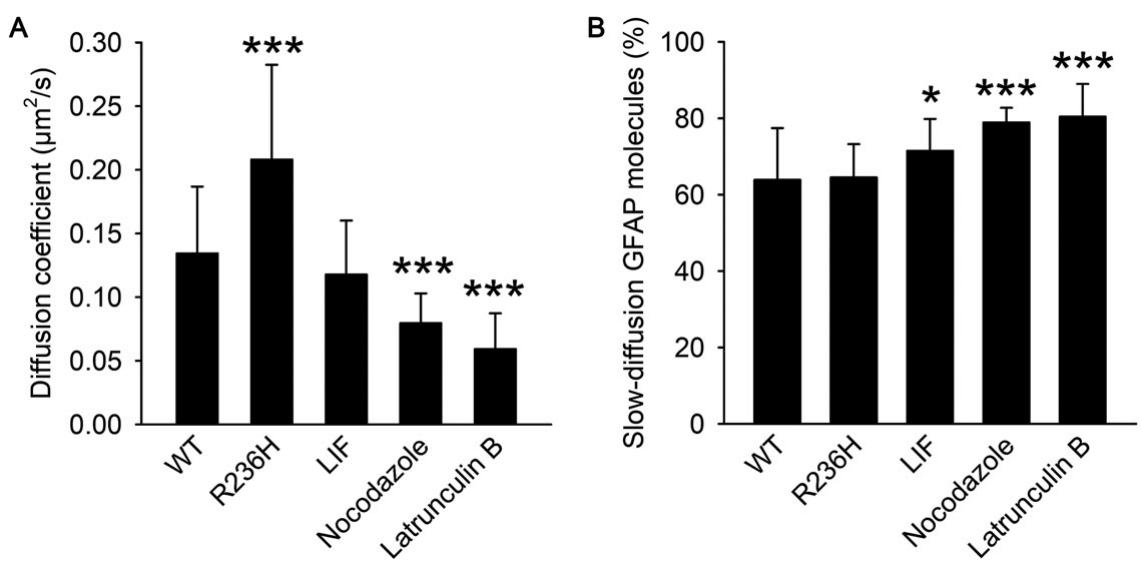

Figure 3. Genetic or pharmacological interference with the diffusion properties of mGFAP molecules. Average diffusion coefficient of rapid-diffusion molecules (A) and fraction of slow-diffusion GFAP molecules (B) calculated for individual cells (20 cells/group) by means of the Spot-On online tool (https://spoton.berkeley.edu/SPTGUI/docs/latest). The AxD-related mutation R236H or incubation of cells with Leukemia Inhibitory Factor (LIF, $100 \mathrm{ng} / \mathrm{ml}$ ), Nocodazole (10 $\mu$ M) or Latrunculin B $(10 \mu \mathrm{M})$ for 2 hours changed the diffusion properties of single mGFAP molecules. LIF is a cytokine that induces the expression and polymerization of GFAP; Nocodazole is a drug that interferes with the formation of microtubules; and Latrunculin $B$ is a drug that disrupts actin filaments. *, significant versus WT, $p<0.05 ;{ }^{\star \star \star} p<0.001$.

construct is more sensitive to the biological background and does not aggregate when mGFAP is mutated, but it allows a wider spectrum of applications, including super-resolution, single-molecule and protein-protein interaction analyses in living cells. We hope these new tools help the GFAP and astrocyte community to advance our understanding of physiological and pathological functions of this intermediate filament in living cells.

Acknowledgements. The authors thank the Advanced Imaging Unit from Gulbenkian Science Institute for support with bioimaging and flow cytometry. The pEGFP-N3-mGFAP plasmid was a kind gift from Dr. Cécile Leduc (Institut Pasteur, Paris, France), the pcDNA-hGFAP construct was a generous gift by Dr. Michael Brenner (University of Alabama, Birmingham, USA), and the template transposon construct with kanamycin resistance and EGFP was kindly provided by Dr. Mika Ruonala. The anti-amyloidogenic compounds J147 and CNB001 were kindly provided by Dr. David Schubert (The Salk Institute, La Jolla, CA, USA). The photoactivatable Janelia Fluor 549 nm (PA-JF549) HaloTag ligand was a kind gift from Dr.Luke Lavis (HHMI's Janelia research campus, Ashburn, VA, USA).

Author contributions. RLV and RQ had equal contribution to this work. RLV developed the EGFP-hGFAP construct and carried out the experiments related to this construct (formation of aggregates and prevention by anti-amyloidogenic drugs). RQ developed the mGFAP-Halo construct and carried out the experiments related to this construct (single-molecule and SRRF analyses). Both of them worked in close collaboration at all cloning and mutagenesis steps. FM tested the formation of GFAP fibrils in different cellular contexts. Alvaro Crevenna and Zach Hensel supervised imaging experiments by RQ and the subsequent data analysis. FH supervised experiments by RLV and RQ, helped with statistical analysis and coordinated the team. FH and RQ wrote the main draft, with contributions from $\mathrm{ZH}$ and RLV.

Funding information. FH was supported by national funds through Fundação para a Ciência e Tecnologia (FCT, Ref. IF/00094/2013/CP1173/CT0005 and PTDC/MED-NEU/31417/2017). FH, ZH and AC were supported by Project LISBOA01-0145-FEDER-007660 (Cellular Structural and Molecular Microbiology) funded by FEDER funds through COMPETE2020 -

JF647 Halo ligand (100 nM) prior to imaging. WT hGFAP or mGFAP assembled into filaments (left panels), but AxD mutations changed this pattern (right panels). hGFAP R239C produced protein aggregates in the cytoplasm, while mGFAP R236H formed a homogeneous pattern throughout the cytoplasm without apparent filament structures. B-C, Quantification of the various patterns observed in U251 cells transfected with (B) WT EGFP-hGFAP (black bars) and mutant EGFP-hGFAP (white bars) or (C) the equivalent mGFAP-Halo constructs. ${ }^{\star \star}$, significant vs WT, $p<0.01 ;{ }^{\star \star \star}, p<0.001$. D, Quantification of U251 cells transfected with the EGFP-hGFAP R239C mutant that displayed a normal filamentous network (black bars) or aggregates (white bars) after treatment with increasing concentrations $(0.1-10 \mu \mathrm{M})$ of the neuroprotective compound CNB-001. All groups were statistically significant versus WT EGFP-hGFAP, $p<0.001$. \#, significant vs EGFP-hGFAP R239C, $p<0.05$; \#\#\#, $p<0.001$. E, Western blots showing similar levels of expression in U251 cells transfected with hGFAP-EGFP and mGFAP-Halo constructs. 
Programa Operacional Competitividade e Internacionalização (POCI). RLV and FM were supported by fellowships from FCT (Refs. PD/BD/128163/2016 and SFRH/BD/133220/2017, respectively).

Data availability statement. The data that support the findings of this study will be made available in a public repository upon acceptance of the manuscript.

Conflicts of interest. The authors declare that they have no conflicts of interests.

\section{Abbreviations:}

hGFAP human glial fibrillary acidic protein;

mGFAP mouse glial fibrillary acidic protein;

EGFP enhanced green fluorescent protein;

AxD Alexander disease;

LIF Leukemia Inhibitory Factor;

SRRF Super-Resolution Radial

Fluctuations.

\section{References}

Anderson, M. A., Ao, Y., \& Sofroniew, M. V. (2014). Heterogeneity of reactive astrocytes. Neurosci Lett, 565, 23-29. https:// doi.org/10.1016/j.neulet.2013.12.030

Bachetti, T., Caroli, F., Bocca, P., Prigione, I., Balbi, P., Biancheri, R., Filocamo, M., Mariotti, C., Pareyson, D., Ravazzolo, R., \& Ceccherini, I. (2008). Mild functional effects of a novel GFAP mutant allele identified in a familial case of adult-onset Alexander disease. Eur J Hum Genet, 16(4), 462-70. doi: 10.1038/sj.ejhg.5201995.

England CG, Luo H, \& Cai W. (2015) HaloTag technology: a versatile platform for biomedical applications. Bioconjug Chem, 26 (6), 975-86. doi: 10.1021/acs.bioconjchem.5b00191.

Gustafsson, N., Culley, S., Ashdown, G., Owen, D. M., Pereira, P. M., \& Henriques, R. (2016). Fast live-cell conventional fluorophore nanoscopy with ImageJ through super-resolution radial fluctuations. Nat Commun, 7(1), 12471. https://doi.org/ 10.1038/ncomms 12471

Herrera F, Chen Q, Fischer WH, Maher P, \& Schubert DR. (2009) Synaptojanin-1 plays a key role in astrogliogenesis: possible relevance for Down's syndrome. Cell Death Differ, 16(6), 910-20. doi: 10.1038/cdd.2009.24.

Hsiao, V. C., Tian, R., Long, H., Der Perng, M., Brenner, M., Quinlan, R. A., \& Goldman, J. E. (2005). Alexander-disease mutation of GFAP causes filament disorganization and decreased solubility of GFAP. J Cell Sci, 118(9), 2057-2065. https:// doi.org/10.1242/jcs.02339

Li D, Liu X, Liu T, Liu H, Tong L, Jia S, Wang YF. (2019) Neurochemical regulation of the expression and function of glial fibrillary acidic protein in astrocytes. Glia, doi: 10.1002/glia.23734. [Epub ahead of print] Review.

Liu, Y., Dargusch, R., Maher, P., \& Schubert, D. (2008). A broadly neuroprotective derivative of curcumin. J Neurochem, 105 (4), 1336-1345. https://doi.org/10.1111/j.1471-4159.2008.05236.x

Messing, A. (2018). Alexander disease. In Handbook of clinical neurology, (Vol. 148, pp. 693-700). https://doi.org/10.1016/ B978-0-444-64076-5.00044-2

Middeldorp, J., \& Hol, E. M. (2011). GFAP in health and disease. Prog Neurobiol, 93(3), 421-443. https://doi.org/10.1016/ j.pneurobio.2011.01.005

Mignot, C., Delarasse, C., Escaich, S., Della Gaspera, B., Noé, E., Colucci-Guyon, E., ... Pham-Dinh, D. (2007). Dynamics of mutated GFAP aggregates revealed by real-time imaging of an astrocyte model of Alexander disease. Exp Cell Res, 313(13), 2766-2779. https://doi.org/10.1016/j.yexcr.2007.04.035

Perng, M.-D., Wen, S.-F., Gibbon, T., Middeldorp, J., Sluijs, J., Hol, E. M., \& Quinlan, R. A. (2008). Glial fibrillary acidic protein filaments can tolerate the incorporation of assembly-compromised GFAP-delta, but with consequences for filament organization and alphaB-crystallin association. Mol Biol Cell, 19(10), 4521-4533. https://doi.org/10.1091/mbc.e08-03-0284

Sheridan DL, Berlot CH, Robert A, Inglis FM, Jakobsdottir KB, Howe JR, Hughes TE. (2002) A new way to rapidly create functional, fluorescent fusion proteins: random insertion of GFP with an in vitro transposition reaction. BMC Neurosci, 19, 3:7.

Tulyeu J, Tamaura M, Jimbo E, Shimbo H, Takano K, Iai M, Yamashita S, Goto T, Aida N, Tokuhiro E, Yamagata T, \& Osaka H. (2019) Aggregate formation analysis of GFAPR416W found in one case of Alexander disease. Brain Dev, 41(2), 195-200. doi: 10.1016/j.braindev.2018.08.009.

Cite this article: Letra-Vilela R, Quiteres R, Murtinheira F, Crevenna A, Hensel Z, Herrera F (2020). New tools for the visualization of glial fibrillary acidic protein in living cells. Experimental Results, 1, e4, 1-8. https://doi.org/10.1017/exp.2020.1 


\section{Peer Reviews}

\section{Reviewing editor: Dr. Michael Nevels}

University of St Andrews, Biomolecular Sciences Building, Fife, United Kingdom of Great Britain and Northern Ireland, KY16 9ST

This article has been accepted because it is deemed to be scientifically sound, has the correct controls, has appropriate methodology and is statistically valid, and met required revisions.

\section{doi:10.1017/exp.2020.1.pr1}

\section{Review 1: New tools for the visualization of glial fibrillary acidic protein in living cells}

Reviewer: Albee Messing

University of Wisconsin Madison

Date of review: 9 December 2019

Published online:

Conflict of interest statement. Reviewer declares none.

Comments to the Author: Previous tagged versions of GFAP have mixed reputations, with some arguing that the tags interfere with normal function. These new versions may offer better options. But exactly where in the hGFAP sequence does the tag reside? Could the difference in aggregation between the two constructs derive from different levels of expression?

\section{Score Card}

\section{Presentation}

Is the data presented in the most useful manner? (40\%)

Does the paper cite relevant and related articles appropriately? (30\%)

\section{Context}

Does the abstract correctly embody the content of the article? (25\%)

Does the introduction give appropriate context? (25\%)

Is the objective of the experiment clearly defined? (25\%)

Are the limitations of the experiment as well as the contributions of the experiment clearly outlined? $(20 \%)$ 
Review 2: New tools for the visualization of glial fibrillary acidic protein in living cells

Reviewer: Dr. Yu-Feng Wang

Date of review: 12 December 2019

Published online:

Conflict of interest statement. No

Comments to the Author: GFAP is not only a structural protein but also a functional molecule. However, evidence supporting a dynamic interaction between GFAP and other molecules remains to be collected in health and disease. The preparation of halo-tagged GFAP is clearly a powerful tool for exploring the mechanisms underlying GFAP plasticity under physiological and pathological conditions. Certainly, readers may expect to see full investigation and discussion of the molecular mechanisms for the differential expressions of the tagged GFAP under different conditions. That needs to consider not only potential spatial obstruction of the tags for the formation of GFAP dimers, tetramers and the filaments, but also for the cellular environments that are responsible for GFAP expression (see review by Li et al, 2019, Glia).

\section{Score Card}

Presentation

\section{0}

Is the article written in clear and proper English? (30\%)

Is the data presented in the most useful manner? (40\%)

Does the paper cite relevant and related articles appropriately? (30\%)

Does the title suitably represent the article? (25\%)

5.0

Does the abstract correctly embody the content of the article? (25\%)

Does the introduction give appropriate context? (25\%)

Is the objective of the experiment clearly defined? (25\%)
Analysis

Does the discussion adequately interpret the results presented? (40\%)

Is the conclusion consistent with the results and discussion? (40\%)

Are the limitations of the experiment as well as the contributions of the experiment clearly outlined? $(20 \%)$ 